\title{
Endocarditis due to Bartonella quintana, the etiological agent of trench fever
}

\author{
Carl Boodman MD, Terence Wuerz MD MSc (Epi), Philippe Lagacé-Wiens MD
}

- Cite as: CMAJ 2020 December 7;192:E1723-6. doi: 10.1503/cmaj.201170

CMAJ Podcasts: author interview at www.cmaj.ca/lookup/doi/10.1503/cmaj.201170/tab-related-content

A 48-year-old man presented to the emergency department with a 2-day history of pleuritic chest pain and shortness of breath. His medical history included HIV infection, diagnosed 14 years earlier in the context of intravenous drug use. Three months previously, he had an undetectable viral load and a CD4 count of 94 cells $/ \mathrm{mm}^{3}$ (normal range: $500-$ 1400 cells $/ \mathrm{mm}^{3}$ ) or 0.09 (normal range $\left.0.50-1.40\right) \times 10^{9} / \mathrm{L}$. The patient adhered to his prescribed antiretroviral regimen (darunavir, ritonavir and abacavir-lamivudine) and prophylaxis against opportunistic infections (valacyclovir, trimethoprim-sulfamethoxazole and fluconazole). In addition, the patient had a congenital solitary kidney with normal baseline renal function, alcohol exposure in utero and alcohol use disorder. He had stopped injecting drugs many years earlier. On several occasions over the past 18 months, the patient had sought care for episodes of chest pain and body lice infestation. Although the patient lived in supportive housing at the time of presentation, he had lived in a homeless shelter less than a year earlier.

On presentation, the patient's temperature was $36.7^{\circ} \mathrm{C}$, blood pressure was $106 / 65 \mathrm{~mm} \mathrm{Hg}$, pulse was 83 beats/min, respiratory rate was 60 breaths/min and oxygen saturation was $100 \%$ on $15 \mathrm{~L} / \mathrm{min}$ of oxygen. On auscultation, he had a holosystolic murmur and an early diastolic decrescendo murmur. Examination of the patient's skin showed hemorrhagic puncta and scabs from arthropod bites (Figure 1). There were no peripheral manifestations of endocarditis.

Computed tomography of the chest and abdomen showed pulmonary emboli, splenic infarcts, splenomegaly and aneurysms of the abdominal aortic branches. A bedside echocardiogram showed severe mitral regurgitation and a large echogenic mitral vegetation. Two sets of blood samples were drawn for cultures before empiric administration of piperacillin-tazobactam and vancomycin for presumptive endocarditis.

The patient's initial tachypnea improved with pain control and his supplemental oxygen was weaned to $2 \mathrm{~L} / \mathrm{min}$. However, 10 hours after presentation, the patient became acutely tachypneic and hypoxemic, requiring $15 \mathrm{~L} / \mathrm{min}$ oxygen to maintain normal saturation. Because of his worsening respiratory distress, we started mechanical ventilation and transferred him to the intensive care unit. A transesophageal echocardiogram showed severe mitral regurgitation with a $25 \times 9 \mathrm{~mm}$ vegetation on the

\section{KEY POINTS}

- Bartonella quintana, the causal agent of trench fever, is transmitted by body lice (Pediculus humanus var. corporis).

- Although B. quintana is notorious for causing disease in the First World War, outbreaks of trench fever have recently occurred in urban populations experiencing homelessness.

- B. quintana causes culture-negative endocarditis and may be fatal without antimicrobial and surgical treatment, despite mild symptomatology during chronic bacteremia. Consultation with infectious disease specialists is encouraged.

- Because B. quintana evades identification in routine blood cultures, diagnosis of $B$. quintana requires serology and explicit communication with the microbiology laboratory to incorporate measures to specifically recover $B$. quintana and to arrange molecular testing.

mitral valve and severe aortic regurgitation associated with multiple smaller vegetations.

Four days after admission, our patient underwent mitral and aortic valve replacement surgery for severe valvular damage. Pathology results showed 4+ polymorphonuclear cells in the tissue of both valves; no organisms were seen on Gram staining, and no pathogen growth was detected on aerobic, anaerobic and fungal cultures. Four blood samples, 2 drawn before antibiotic administration, were culture-negative after 5 days of incubation. We were not able to culture any organism despite lysis centrifugation and prolonged incubation of 6 weeks. We obtained serologies for Bartonella quintana, Bartonella henselae, Coxiella burnetii and Brucella species as recommended when there is concern for culture-negative endocarditis. Serologies for both Bartonella quintana and Bartonella henselae showed immunoglobulin $\mathrm{G}$ titres greater than 1:8192 (normal range < 1:64), ${ }^{1}$ while those for Coxiella and Brucella were negative. Results from 16S rRNA sequencing from both valves showed Bartonella quintana genetic material with 100\% homology over 741 base pairs.

Nine days after admission, we changed the antimicrobial therapy to doxycycline and gentamicin. Because of worsening renal failure, we changed gentamicin to ceftriaxone after 4 days. Ceftriaxone and doxycycline were continued for 


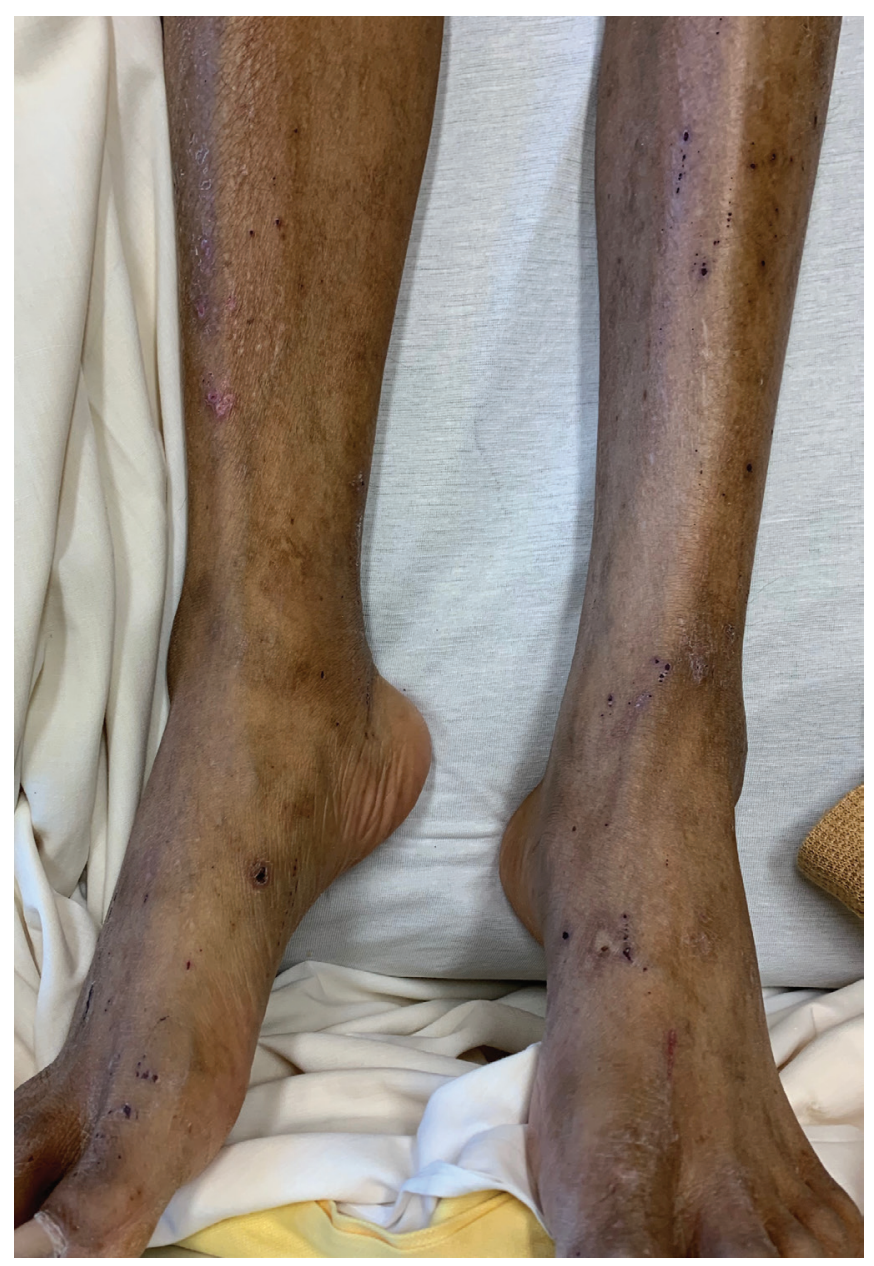

Figure 1: Hemorrhagic punctate lesions and scabs from ectoparasitosis on a 48-year-old man with endocarditis due to Bartonella quintana (patient 1). Note that, unlike bedbugs, the lesions do not follow areas of pressure such as belt or sock lines.

6 weeks after surgery. Two weeks after valve replacement, the patient was discharged from hospital and completed treatment as an outpatient. He remained well at follow-up 3 months after discharge.

Three additional patients with $B$. quintana were identified in Winnipeg over a 6-month period, all of whom had accessed resources at the same homeless shelter (details in Appendix 1 and Appendix 2, available at www.cmaj.ca/lookup/doi/10.1503/ cmaj.201170/tab-related-content). A summary of all 4 cases is presented in Box 1 .

\section{Discussion}

Bartonella quintana is transmitted via the inoculation of infected body lice feces into breaks in the skin. ${ }^{2,3}$ Historically, the pathogen has been associated with trench fever during the First World War, but urban outbreaks among individuals who experience homelessness have been described since the 1990s in the United States and France. ${ }^{1,4,5}$ Seroprevalence for Bartonella can exceed $20 \%$ among people experiencing homelessness who have a severe alcohol use disorder or who use intravenous drugs. ${ }^{1,4,5}$
B. quintana endocarditis is associated with a $10 \%-12 \%$ mortality rate despite targeted antimicrobial therapy and valvular surgery. ${ }^{3,6}$ We are aware of only 4 other cases of $B$. quintana previously reported in Canada. ${ }^{7,8}$

\section{Clinical presentation}

Trench fever is a clinical syndrome caused by B. quintana, associated with fever, headache and malaise. ${ }^{3,6,9}$ Splenomegaly and bone pain localized to the anterior tibia have been described. ${ }^{2}$ Trench fever was originally described as "quintan fever" because of its episodic symptom recurrence every 5 days, leading to the species designation B. quintana. ${ }^{6}$ Although prolonged bacteremia over many months may be associated with minimal symptoms and no fever, it may also lead to endovascular disease such as culture-negative endocarditis and mycotic aneurysms. ${ }^{5,6,9}$

The patient described here had repeatedly sought care for chest pain and body lice infestation, also known as ectoparasitosis, before the diagnosis of $B$. quintana endocarditis. The patient's indolent presentation, until the development of severe embolic disease, is consistent with B. quintana's prolonged bacteremia and mild symptomatology. ${ }^{3,9}$

\section{Diagnosis}

B. quintana is a fastidious, gram-negative, intraerythrocytic bacillus. Prolonged incubation of up to 6 weeks may be necessary to isolate this organism in blood cultures. ${ }^{3,8,9}$ Considering that microbiology laboratories routinely maintain blood cultures for 5 days, it is essential to discuss cases of suspected $B$. quintana with the microbiology laboratory to extend incubation and to perform additional techniques to release intracellular bacteria and improve yield, such as lysis centrifugation or freeze-thaw. ${ }^{8,9}$ Serological testing may show exposure to B. quintana, and elevated titres are associated with endocarditis, as seen here. ${ }^{6,9}$ Titres over 1:800 are consistent with endocarditis, and chronic infection without endocarditis is associated with lower titres. ${ }^{6}$ However, serology is prone to cross-reactivity, especially with Bartonella henselae, the causative pathogen of cat scratch disease, and chronic bacteremia without endocarditis may be associated with low titres., When B. quintana serology is requested in Canada, serum samples are sent to the National Microbiology Laboratory in Winnipeg, the results of which take about 1 month to return.

Molecular testing with 16S rRNA sequencing of valvular tissue provides species-level diagnosis of $B$. quintana endocarditis. Although high levels of genetic similarity between certain Bartonella species may interfere with their identification by $16 \mathrm{~S}$ sequencing, the valvular tissue obtained here showed $100 \%$ homology with $B$. quintana and substantial separation from other species, including $B$. henselae. A difference of greater than $0.8 \%$ from the species with the next highest $16 \mathrm{~S}$ homology is sufficient to achieve species discrimination. In this case, the species with the next highest homology (99.03\%) was Bartonella senegalensis, which is not a human pathogen.

B. quintana infection may be diagnosed without molecular species-level confirmation when no valve tissue is obtained. In 
Box 1: Summary of Bartonella quintana infections among 4 patients in Winnipeg

\begin{tabular}{|c|c|c|c|c|}
\hline Characteristic & Patient $1^{\star}$ & Patient 2 & Patient 3 & Patient 4 \\
\hline Age, yr & 48 & 62 & 35 & 33 \\
\hline Gender & Male & Male & Male & Male \\
\hline Presenting syndrome & $\begin{array}{l}\text { Embolization and } \\
\text { valvulopathy }\end{array}$ & $\begin{array}{l}\text { Murmur } \\
\text { (incidental) }\end{array}$ & $\begin{array}{l}\text { Intracranial hemorrhage } \\
\text { (ruptured mycotic } \\
\text { aneurysm) }\end{array}$ & $\begin{array}{l}\text { Intracranial hemorrhage } \\
\text { (ruptured mycotic } \\
\text { aneurysm) }\end{array}$ \\
\hline Valvular involvement & Mitral and aortic & Mitral & Mitral & Mitral \\
\hline Previous valvular disease & None & None & Rheumatic heart disease & None \\
\hline Diagnostic testing & $\begin{array}{l}\text { Serology and } 16 \mathrm{~S} \text { from } \\
\text { valve tissue }\end{array}$ & $\begin{array}{l}\text { Serology and } 16 \mathrm{~S} \text { from } \\
\text { valve tissue }\end{array}$ & Serology & Serology \\
\hline Surgical intervention & Valve replacement & Valve replacement & Cerebral artery coiling & No intervention \\
\hline Possible exposure & Slept at homeless shelter & Slept at homeless shelter & $\begin{array}{l}\text { Slept at homeless shelter, } \\
\text { possible ectoparasitosis } \\
\text { in rural community }\end{array}$ & $\begin{array}{l}\text { Received meals at } \\
\text { homeless shelter, } \\
\text { possible ectoparasitosis } \\
\text { in rural community }\end{array}$ \\
\hline Links to rural community & No & No & Yes & Yes \\
\hline $\begin{array}{l}\text { Emergency department } \\
\text { presentation within } 3 \text { months } \\
\text { before hospital admission }\end{array}$ & Yes & Yes & No & Yes \\
\hline Antimicrobial therapy & $\begin{array}{l}\text { - Gentamicin (4d) } \\
\text { - Ceftriaxone (42 d) } \\
\text { - Doxycycline (42 d) }\end{array}$ & $\begin{array}{l}\text { - Gentamicin (14 d) } \\
\text { - Doxycycline (42 d) }\end{array}$ & $\begin{array}{l}\text { - Ceftriaxone (43 d) } \\
\text { - Vancomycin }(21 \mathrm{~d}) \\
\text { - Daptomycin (22 d) }\end{array}$ & $\begin{array}{l}\text { - Ceftriaxone }(56 \mathrm{~d}) \\
\text { - Vancomycin (56 d) } \\
\text { - Doxycycline }(56 \mathrm{~d}) \\
\text { - Gentamicin (14 d) }\end{array}$ \\
\hline Outcome & Full recovery & Full recovery & $\begin{array}{l}\text { Mild neurologic deficits } \\
\text { (mild expressive aphasia) }\end{array}$ & $\begin{array}{l}\text { Severe neurologic deficits } \\
\text { (paralysis and severe } \\
\text { expressive aphasia) }\end{array}$ \\
\hline
\end{tabular}

such cases, it is important to confirm possible exposure to body lice and to exclude substantial exposure to cats, which harbour $B$. henselae that may cause serologic cross-reactivity with $B$. quintana. Considering the rarity of requests for B. quintana testing, infections due to $B$. quintana likely remain underdiagnosed.

\section{Treatment and prevention}

Standard treatment of $B$. quintana bacteremia and endocarditis includes a combination of gentamicin and doxycycline. ${ }^{6}$ Gentamicin is typically administered for 14 days, with doxycycline. Doxycycline is continued for 4 weeks in the absence of endocarditis and for at least 6 weeks in cases of endocarditis. ${ }^{6}$ The inclusion of gentamicin is associated with improved clinical outcomes and clearance of bacteremia. ${ }^{6}$ However, given the risk of gentamicin toxicity, cases have been successfully treated with regimens of ceftriaxone and either doxycycline or rifampin. ${ }^{6-8,10}$ In our case, the patient was treated with a regimen of ceftriaxone without prolonged aminoglycoside administration, with good clinical outcomes. Further studies are necessary to support ceftriaxone and doxycycline regimens without the addition of aminoglycosides. We strongly suggest consultation with infectious disease specialists. As illustrated in our patient, trimethoprim-sulfamethoxazole does not treat or prevent infections with $B$. quintana. A low threshold to offer therapy to address body lice and other ectoparasite infestations should be considered to prevent $B$. quintana infection among at-risk populations.

\section{Conclusion}

B. quintana, transmitted by body lice, causes trench fever and can lead to culture-negative endocarditis among people who experience homelessness. Clinicians should consider Bartonella serology, echocardiography and infectious disease consultation when caring for individuals who present unwell with a history of body lice infestation. $B$. quintana infection likely remains underdiagnosed. Seroprevalence, bacteremia surveillance and ectoparasite studies are needed to understand its true burden among people who experience homelessness.

\section{References}

1. Jackson LA, Spaeh DH, Kippen DA, et al. Seroprevalence to Bartonella quintana among patients at a community clinic in downtown Seattle. J Infect Dis 1996;173:1023-6.

2. OhI ME, Spach DH. Bartonella quintana and urban trench fever. Clin Infect Dis 2000;31:131-5.

3. Raoult D, Foucault C, Brouqui P. Infections in the homeless. Lancet Infect Dis 2001;1:77-84.

4. Cormer JA, Flynn C, Regnery RL, et al. Antibodies to Bartonella species in inner-city intravenous drug users in Baltimore, Md. Arch Intern Med 1996; 156:2491-5. 
5. Brouqui P, Houpikian P, Dupont HT, et al. Survey of the seroprevalence of Bartonella quintana in homeless people. Clin Infect Dis 1996;23:756-9.

6. Foucault C, Brouqui P, Raoult D. Bartonella quintana characteristics and clinical management. Emerg Infect Dis J 2006;12:217-23.

7. Lam JC, Fonseca K, Pabbaraju K, et al. Bartonella quintana endocarditis outside of the Europe-African gradient: comprehensive review of cases within North America. Am J Trop Med Hyg 2019;100:1125-9.
8. Keynan Y, Mackenzie L, Lagacé-Wiens P. Quintessential culture-negative endocarditis. Can J Cardiol 2016;32:395.e9-10.

9. Brouqui $\mathrm{P}$, Lascola $\mathrm{B}$, Roux V, et al. Chronic Bartonella quintana bacteremia in homeless patients. N Engl J Med 1999;340:184-9.

10. Raoult D, Fournier P-E, Vandenesch F, et al. Outcome and treatment of Bartonella endocarditis. Arch Intern Med 2003;163:226-30.

\section{Competing interests: None declared.}

This article has been peer reviewed.

The authors have obtained patient consent.

Affiliations: Section of Infectious Diseases (Boodman, Wuerz), Department of Internal Medicine and Departments of Medical Microbiology and Infectious Diseases (Boodman, Lagacé-Wiens) and Community Health Sciences (Wuerz), Max Rady College of Medicine, University of Manitoba; Shared Health (Lagacé-Wiens), Winnipeg, Man.
Contributors: Carl Boodman contributed to the overall conception of the article, drafted the manuscript and provided specific details of the clinical case. Philippe Lagacé-Wiens provided microbiologic support to the case. Terence Wuerz provided clinical advice and supervision during the cases. All authors provided essential manuscript edits and critical revisions before submission. All authors gave final approval of the version to be published and agreed to be accountable for all aspects of the work.
Acknowledgments: The authors thank their patients for their positive outlook, their willingness to participate in this project and their resilience in the face of difficult circumstances.

Correspondence to: Carl Boodman, boodmanc@myumanitoba.ca; Philippe Lagacé-Wiens, plagacewiens@sharedhealthmb.ca 